\title{
Precisão dimensional de modelos obtidos de moldes de alginato: técnica da união de análogos
}

\author{
Cast accuracy obtained from alginate impressions: analogue joining \\ technique \\ Precisión dimensional de modelos obtenidos de moldes de alginato: técnica \\ de unión de análogos \\ Nelson Talatoci OSHIRO FILHO \\ Túlio Marcos Kalife COELHO \\ João Paulo SCHUSSLER \\ Elizeu INSAURRALDE \\ Marco Petry Laureano LEME
}

Faculdade de Odontologia, Universidade Federal do Mato Grosso do Sul (UFMS) 79070-900 Campo Grande-MS, Brasil

\begin{abstract}
Resumo
Objetivo: Esta pesquisa avaliou a estabilidade dimensional de modelos obtidos de moldes de alginato utilizando a técnica de união dos análogos. Materiais e Métodos: foi utilizado um modelo mestre representando uma mandíbula com 4 implantes osseointegrados instalados, sobre o qual foi realizado a moldagem com moldeira aberta dos transferentes unidos por fio metálico rígido e resina acrílica autopolimerizável, utilizando o alginato. Foram feitas 40 moldagens ao todo, sendo 20 para cada grupo. Após as moldagens, os moldes foram vazados utilizando gesso pedra de alta resistência, sendo que em um grupo foi realizada a união de análogos previamente. Após o término da reação de presa do gesso, os modelos foram levados para análise na lupa estereoscópica, onde foram realizadas 3 medições da distância dos análogos entre si, obtendo-se uma média para cada modelo. Resultados: As médias obtidas foram comparadas com a média da distância entre os análogos e enviadas para análise estatística para a obtenção dos resultados, utilizando o teste Tukey HSD. Os resultados mostraram que as medidas de ambos os grupos tiveram diferenças estatísticas em relação ao modelo mestre, sendo que com a união houve alteração nas medidas A e C e sem a união houve alteração nas medidas $\mathrm{A}$, C e D. Conclusão: Concluiu-se que os modelos com maior fidelidade dimensional são os do grupo com a união de análogos. Descritores: Técnica de Moldagem Odontológica; Implantes Dentários; Prótese Dentária.
\end{abstract}

\begin{abstract}
Objective: This study evaluated the accuracy of cast obtained from alginate impressions using analogue joining technique. Materials and Methods: used a model master representing a jaw with 4 dental implants installed, on which perform the technique of open impression tray with copings bound by rigid metal wire and acrylic resin, using alginate. 40 impressions were made, 20 for each group. After molding, the molds were leaked using special plaster, and was performed union of analogs previously in a group. After completion of the reaction of gypsum models were led for analysis on a stereomicroscope, where three measurements were performed of distance between analogs, obtaining an average for each model. Results: Mean values were compared with the mean distance between the analogs and sent to statistical analysis to obtain the results using the Tukey HSD test. The results showed that the measures of both groups had statistically significant differences compared to the master model, and with the union was verified change in measures $\mathrm{A}$ and $\mathrm{C}$ and the without union was verified change in measures $\mathrm{A}, \mathrm{C}$ and $\mathrm{D}$. Conclusion: We conclude that models with higher dimensional fidelity are the group with the union of analogues.
\end{abstract}

Descriptors: Dental Impression Technique; Dental Implants; Dental Prosthesis.

\section{Resumen}

Objetivo: Esta investigación evaluó la estabilidad dimensional de modelos obtenidos de moldes de alginato utilizando la técnica de unión de los análogos. Materiales y Métodos: se utilizó un modelo maestro que representaba una mandíbula con 4 implantes oseointegrados instalados, sobre el cual se realizó el moldeado con marco abierto de los transferentes unidos por hilo metálico rígido y resina acrílica autopolimerizable, utilizando el alginato. Se realizaron 40 moldes en total, siendo 20 para cada grupo. Después de los moldes, los moldes fueron filtrados utilizando yeso piedra de alta resistencia, siendo que en un grupo se realizó la unión de análogos previamente. Después de la terminación de la reacción de presa del yeso, los modelos fueron llevados para análisis en la lupa estereoscópica, donde se realizaron 3 mediciones de la distancia de los análogos entre sí, obteniéndose una media para cada modelo. Resultados: Los promedios obtenidos fueron comparados con la media de la distancia entre los análogos y enviados para análisis estadísticos para la obtención de los resultados, utilizando la prueba Tukey HSD. Los resultados mostraron que las medidas de ambos grupos tuvieron diferencias estadísticas con respecto al modelo maestro, siendo que con la unión hubo alteración en las medidas A y C y sin la unión hubo alteración en las medidas A, C y D. Conclusión: Se concluyó que los modelos con mayor fidelidad dimensional son los del grupo con la unión de análogos.

Descriptores: Técnica de Impresión Dental; Implantes Dentales; Prótesis Dental.

\section{INTRODUÇÃO}

Um sistema estomatognático íntegro e saudável é evidentemente essencial para um bom comportamento social, uma vez que pessoas com deficiência na arcada dentária não são prejudicadas somente nas funções mastigatória e sistêmica, mas sim em tudo o que representa um sorriso harmônico. Atualmente pacientes edêntulos são tratados mais comumente por meio de próteses que buscam devolver estética, função e acima de tudo, qualidade de vida. Vários estudos foram feitos até que chegássemos a uma tecnologia capaz de adaptar e fixar as próteses na boca de maneira refinada e conveniente. A osseointegração de implantes permite trabalhos protéticos eficazes na solução de reabilitações orais ${ }^{1}$.

Apesar dos benefícios, os implantes diferem dos dentes naturais quanto a sua implantação, sendo que estes se interpõem ao osso sem o comum arranjo de ligamento periodontal, o que prejudica a capacidade de absorção e distribuição das forças mastigatórias. Por isso, alguns princípios devem ser seguidos à risca, uma vez que o conjunto prótese-implante deve estar unido por uma força de travamento livre de tensões desequilibradas, garantindo a longevidade do tratamento. O sucesso da osseointegração dos implantes está relacionado a um meticuloso planejamento cirúrgicoprotético que proporcione adaptação passiva da prótese e consequentemente uma correta distribuição de cargas. A utilização de técnicas e materiais adequados é indispensável para garantir fidelidade dos modelos de estudo e trabalho ${ }^{2}$.

Alguns dispositivos como transferentes e análogos são utilizados para reproduzir os implantes nos modelos de gesso, porém no momento da adaptação dos análogos pode haver movimentação ou rotação dos transferentes no interior do molde, interferindo na precisão linear de modelos de gesso $^{3}$. 
Em moldagens para implantes múltiplos a utilização de tranferentes quadrados unidos por pequenas barras ou fio dental, associados à resina acrílica, proporciona maior estabilidade e gera modelos de maior precisão em relação à posição dos implantes, e sempre que possível, deve-se utilizar moldeira aberta individual para evitar a rotação durante a rápida remoção do transferente ${ }^{4}$.

Todas as etapas de moldagem devem ser consideradas, inclusive a desinfecção dos moldes que pode ser realizada sem comprometer a estabilidade dimensional dos materiais ${ }^{5}$. Seguir as orientações do fabricante é a melhor maneira de contornar os diversos fatores que levam a distorções. Estudos revelam que alterações dimensionais nos modelos são inevitáveis e podem ser significantes quando se trabalha na ordem de micrometros.

Analisando o comportamento morfodimensional de seis siliconas de adição, um poliéter, um polissulfeto, uma silicona de condensação e um hidrocolóide irreversível e concluiu-se que todos os materiais de moldagem testados produziram modelos de gesso com alterações dimensionais estatisticamente significantes quando comparados com o modelo mestre ${ }^{6}$.

$\mathrm{O}$ uso de um material de moldagem preciso não garante resultado final positivo caso a manipulação do gesso for negligenciada. A qualidade do gesso é determinada por diversos fatores, tais como a presença de sais ou outros componentes na água, o tipo de manipulação e vazamento do modelo, o auxilio ou não de vibrador, a composição do gesso, entre outros ${ }^{7}$. Isso demonstra que na prática clínica e laboratorial a realização de uma técnica exata torna-se difícil pelos inúmeros fatores extrínsecos e intrínsecos a serem considerados $^{8}$. Com base no exposto, o propósito deste trabalho foi avaliar a estabilidade dimensional de modelos obtidos de moldes de alginato utilizando a técnica de união dos análogos.

\section{MATERIAL E MÉTODO}

Neste estudo, foi utilizado um modelo mestre representando uma mandíbula com 4 implantes (Máster Porous - Conexao Sistema de Próteses Ltda., São Paulo SP Brasil) osseointegrados instalados (Figura 1), na fase de moldagem para confecção da prótese. Utilizamos em todos os grupos transferentes (Máster Porous - Conexão Sistema de Próteses Ltda., São Paulo SP, Brasil), para moldeira aberta do tipo dos implantes instalados no modelo mestre, ou seja, hexágono externo com plataforma de $4,1 \mathrm{~mm}$ de diâmetro (Figura 2).

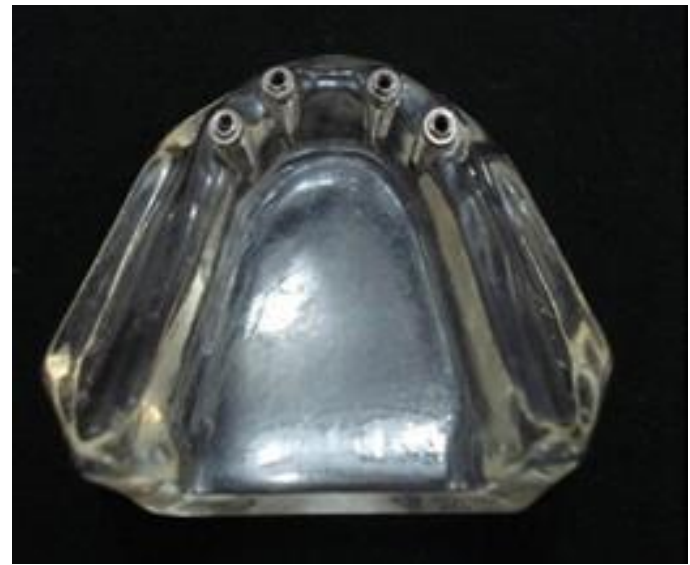

Figura 1: Modelo mestre com 4 implantes regulares.

No Grupo 1, os transferentes foram instalados e após a certeza de sua adaptação foi dado torque manual aos mesmos. Foi feita a união entre os transferentes através do uso de fio metálico rígido (Clipe de Papel 2/0 - ACC Indústria de Artigos Para Escritório S/A, Santo André-SP, Brasil) (Figura 3), alfa-cianocrilato (Super Bonder Flex Gel Control - Loctite, SP, Brasil) (Figura 4) e resina acrílica autopolimerizável (Trim Plus II - Bosworh Company, Skokie-IL, EUA) (Figura 5).

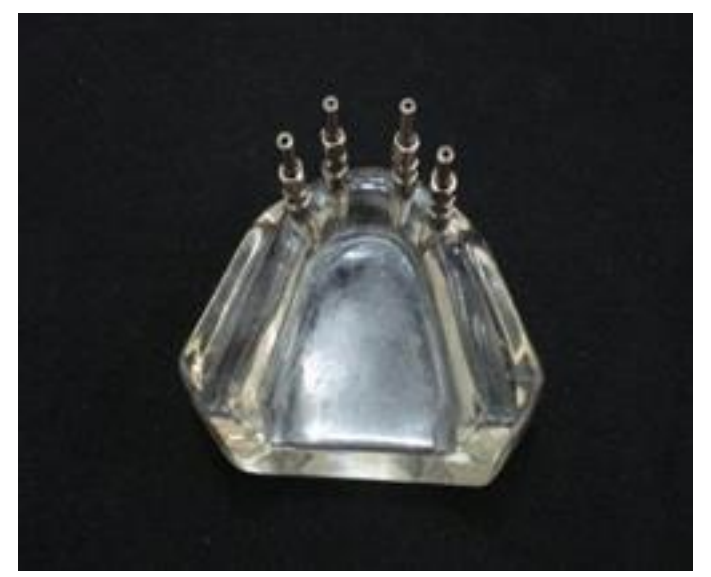

Figura 2: Modelo mestre com transferentes em posição.

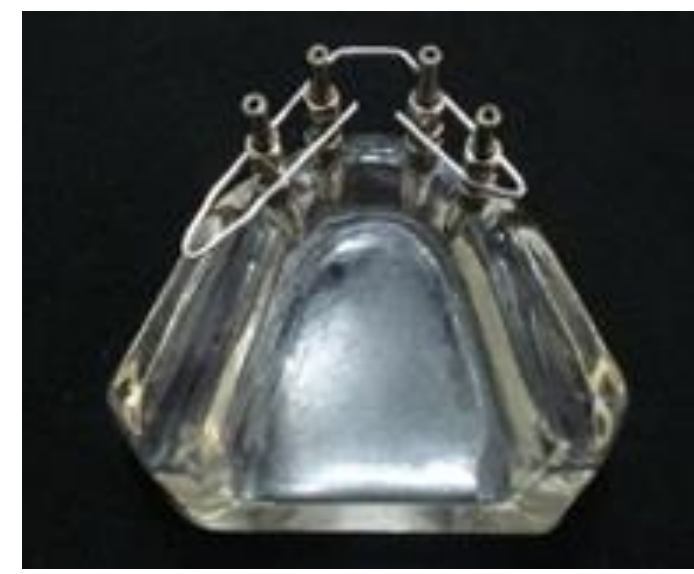

Figura 3: Posicionamento do fio metálico rígido.

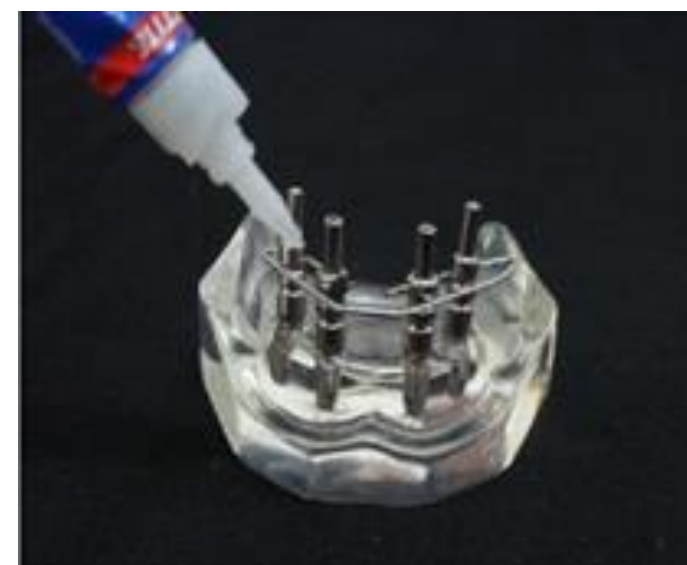

Figura 4: Fixação prévia do fio com alfacianocrilato.

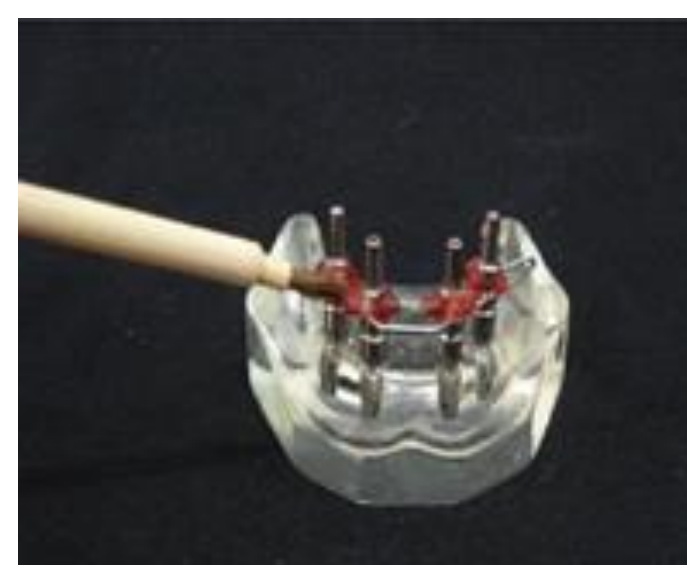

Figura 5: Fixação com resina acrílica autopolimerizável.

Foi aguardada a presa da resina acrílica e subsequentemente realizada a moldagem utilizando uma moldeira plástica de estoque perfurada na região dos transferentes (Figura 6). Foi manipulado o alginato (Cavex ColorChange - Cavex Holland BV, Haarlem, Netherlands) segundo a proporção água/pó recomendada pelo fabricante. Com o auxílio de uma seringa de moldagem o alginato foi colocado ao redor dos transferentes, carregando a moldeira e a levando em posição, para então aguardar o término da reação de presa do mesmo, ou seja, cerca de 2 minutos e 30 segundos. Após a presa do material de moldagem, os transferentes foram soltos do modelo mestre, para retirar o molde e colocar os análogos em posição (Figura 7), realizando em seguida o vazamento dos moldes com gesso pedra de alta resistência (Durone IV - Dentsply Ind. e Com. Ltda, Rio de Janeiro, Brasil) (Figura 8).

O Grupo 2 diferiu do Grupo 1 a partir da etapa de instalação dos análogos, onde após colocá-los em posição, foi realizada a união entre os análogos de maneira semelhante a 
união dos transferentes, por meio do uso de fio metálico rígido, alfacianocrilato e resina acrílica autopolimerizável (Figura 9).

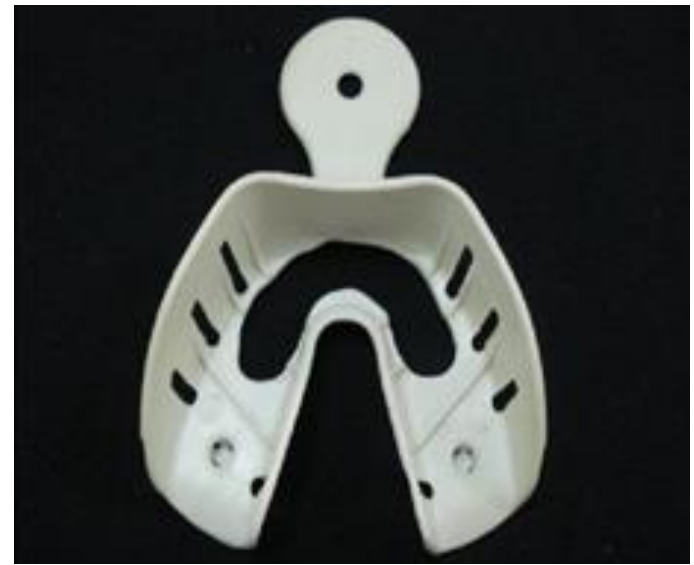

Figura 6: Seleção da moldeira de estoque e confecção da abertura.

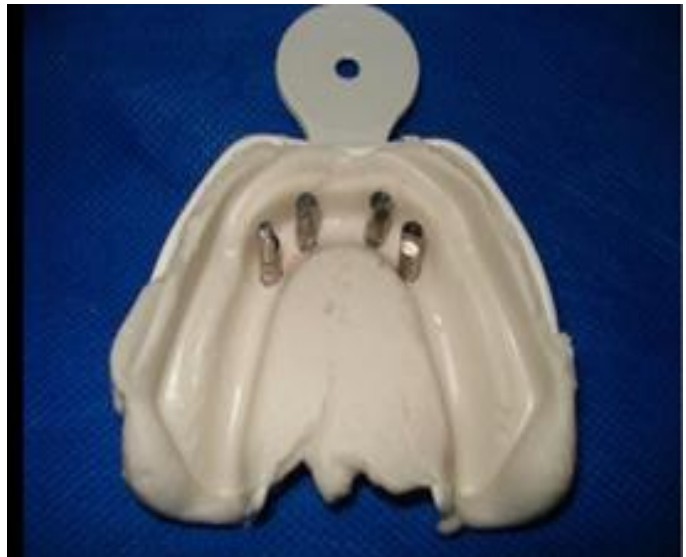

Figura 7: Molde do Grupo 1, com os análogos em posição.

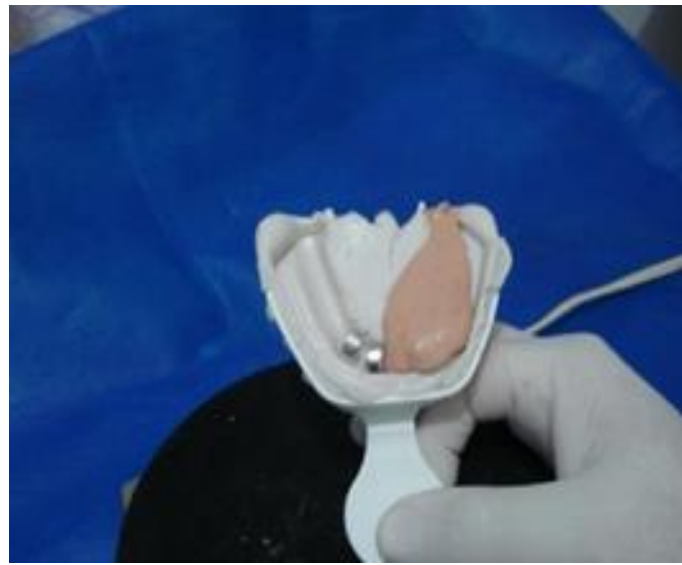

Figura 8: Vazamento do molde com gesso de alta resistência.

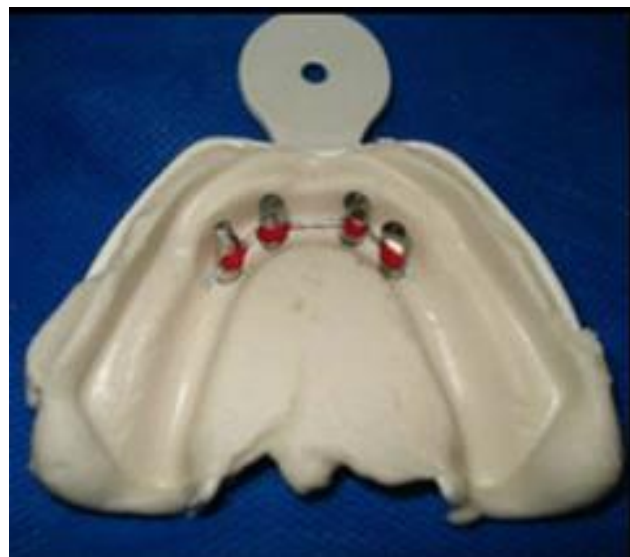

Figura 9: Molde do Grupo 2, com os análogos unidos em posição.

Após a polimerização da resina acrílica os moldes foram vazados com gesso pedra de alta resistência (Figura 10). Foram feitos 40 moldes, sendo $20 \mathrm{em}$ cada Grupo. Atingido o tempo de presa do gesso os modelos (Figura 11) foram levados para análise na lupa estereoscópica (Figura 12), onde foram feitas 3 medições da distância dos análogos entre si, tendo como referência o centro de cada um (Figura 13), obtendo-se uma média das distâncias entre os análogos em cada modelo. As medições foram realizadas por dois analisadores devidamente orientados e que não sabiam a qual grupo correspondia o modelo. As médias obtidas foram comparadas com a média da distância entre os análogos do modelo-mestre. Os resultados obtidos foram submetidos à análise estatística.

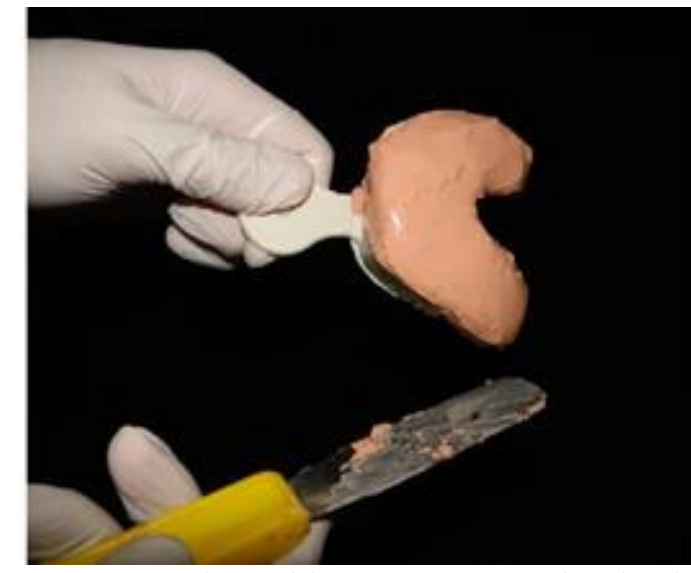

Figura 10: Vazamento do molde com gesso pedra de alta resistência.

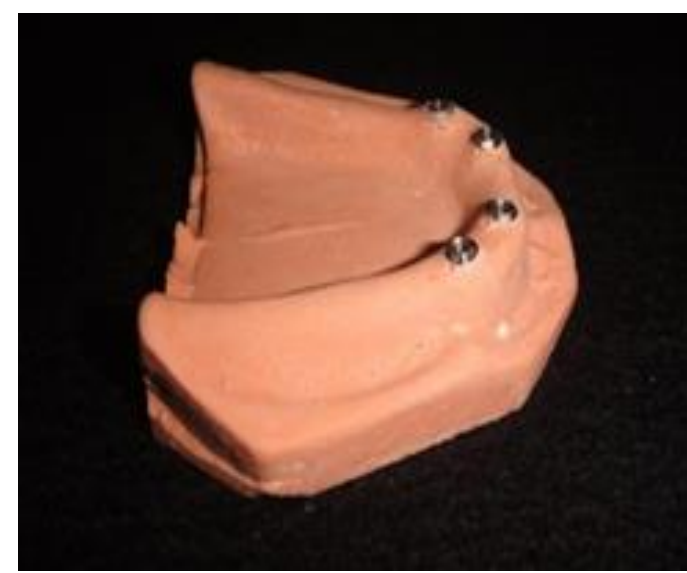

Figura 11: Obtenção do modelo de gesso.

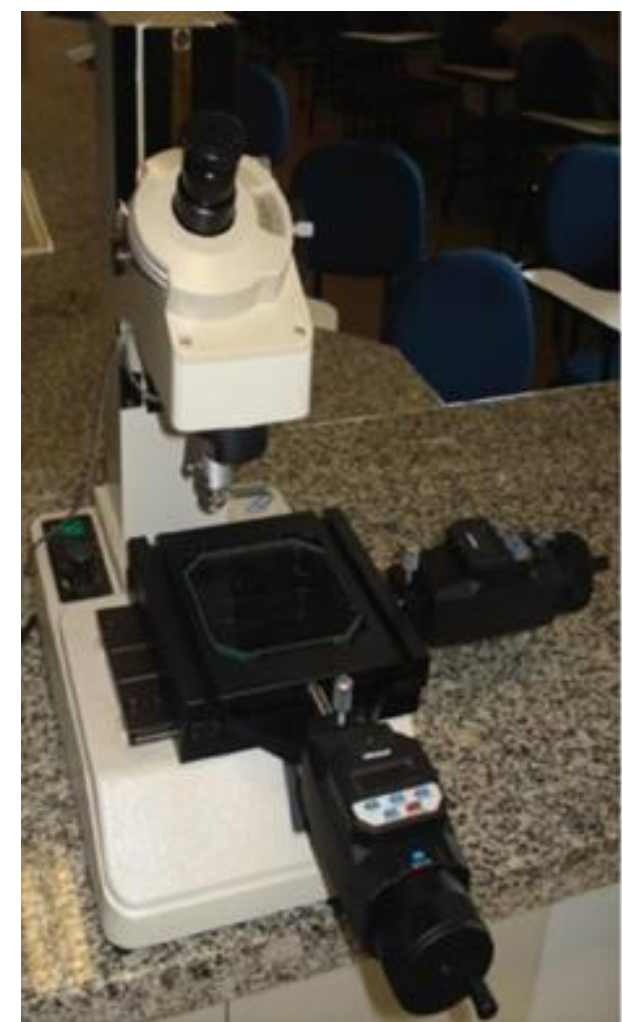

Figura 12: Lupa estereoscópica.

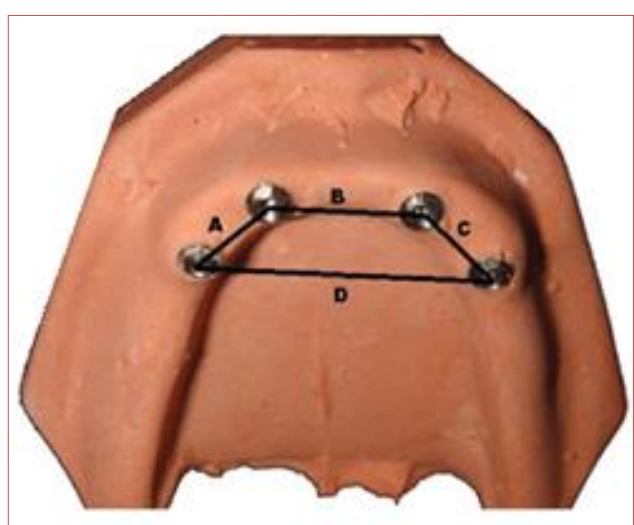

Figura 13: Medidas entre os análogos.

\section{RESULTADOS}

Quando comparado o grupo com e sem união de análogos, todas as medidas apresentam valores diferentes estatisticamente, com $\mathrm{p}<0,05$ pelo teste de Tukey HSD. Quando analisadas as medidas B, C e D, os valores para o uso de alginato com união resultou em valores maiores e diferentes do grupo de alginato sem união (Tabela 1 Alginato com e sem união). Para que se saiba se o fato de um grupo sem união ser diferente de outro com união na mesma medida representa importância em relação à medida padrão 
do modelo mestre, as comparações com o modelo mestre são necessárias.

Tabela 1. Alginato com e sem união- análise descritiva e comparação entre grupos (em destaque: os valores de "p", aqui relatados pela sigla "sig" para cada medida A,B,C,D)

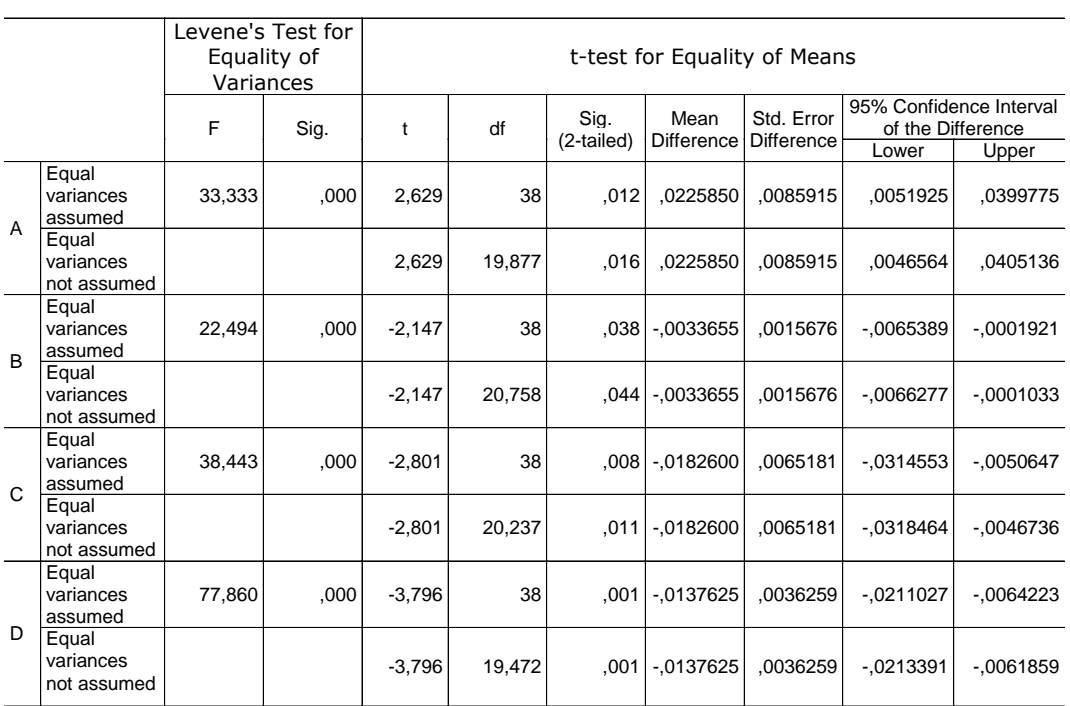

Quando comparadas as medidas sem união de análogos com as do modelo mestre, as medidas A, C e D mostraram que existe diferença estatística entre o modelo mestre e o alginato sem união, com $\mathrm{p}<0,05$ pelo teste de Tukey HSD. Para a medida B os dados mostraram-se semelhantes entre si, com $\mathrm{p}>0,05$ pelo teste de Tukey HSD. Sendo que nas medidas C e D o modelo mestre apresentou valores maiores que os do alginato (Tabela 2 - Alginato sem união e modelo mestre).

Tabela 2: Alginato sem união e modelo mestre- análise descritiva e comparação entre grupos (em destaque: os valores de "p", aqui relatados pela sigla "sig" para cada medida A,B,C,D)

\begin{tabular}{|c|c|c|c|c|c|c|c|c|c|c|}
\hline & \multicolumn{2}{|c|}{$\begin{array}{l}\text { Levene's Test } \\
\text { for Equality } \\
\text { of Variances }\end{array}$} & \multicolumn{7}{|c|}{$\mathrm{t}$-test for Equality of Means } \\
\hline & & tr var & Sig. & \multirow[t]{2}{*}{ t } & \multirow[t]{2}{*}{ df } & 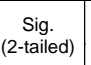 & $\begin{array}{c}\text { Mean } \\
\text { Difference }\end{array}$ & $\begin{array}{l}\text { Std. Error } \\
\text { Difference }\end{array}$ & \multicolumn{2}{|c|}{$95 \%$ Confidence Interval of } \\
\hline & Equal & & & & & & & & Lower & Upper \\
\hline \multirow{2}{*}{ A } & $\begin{array}{l}\text { varaincess } \\
\text { assumed }\end{array}$ & 48,197 & , & 4,328 & 38 & , & , 0367600 & ,0084939 & |0195649 & ,0539551 \\
\hline & $\begin{array}{l}\text { variances not } \\
\text { assumed }\end{array}$ & & & 4,328 & 19,000 & , 000 & ,0367600 & ,0084939 & ,0189820 & ,0545380 \\
\hline \multirow{2}{*}{ B } & $\begin{array}{l}\text { Equal } \\
\text { varainces } \\
\text { assumed }\end{array}$ & 38,448 & ,000 & $-1,971$ & 38 & ,056 &,- 0030205 & ,0015324 & - & ,0000818 \\
\hline & $\begin{array}{l}\text { Equal } \\
\text { varainces not } \\
\text { assumed }\end{array}$ & & & $-1,971$ & 19,000 & ,063 &,- 0030205 & ,0015324 & -0062279 & , 0001869 \\
\hline \multirow{2}{*}{ c } & $\begin{array}{l}\text { Equal } \\
\text { variances } \\
\text { assumed }\end{array}$ & 59,357 & , 000 & $-2,397$ & 38 & ,022 & -0153775 & ,0064145 &,- 0283629 & -0023921 \\
\hline & $\begin{array}{l}\text { Equal } \\
\text { variances not } \\
\text { assumed }\end{array}$ & & & $-2,397$ & 19,000 & ,027 & - -0153775 & ,0064145 & - -0288032 & -0019518 \\
\hline \multirow{2}{*}{ D } & $\begin{array}{l}\text { Equal } \\
\text { varainces } \\
\text { assumed }\end{array}$ & 98,142 & ,000 & $-3,690$ & 38 & ,001 &,- 0132975 & ,0036035 & - -0205925 & -, 0060025 \\
\hline & $\begin{array}{l}\text { Equal } \\
\text { variances not } \\
\text { assumed }\end{array}$ & & & $-3,690$ & 19,000 & ,002 &,- 0132975 & ,0036035 &,- 0208398 & -0057552 \\
\hline
\end{tabular}

Na comparação entre as medidas do modelo mestre e as obtidas com união de análogos houve apenas diferença estastistica $(\mathrm{p}<0,05)$ para as medidas A e C. Para a medida B e D os dados mostraram-se semelhantes entre si, com $\mathrm{p}>0,05$ pelo teste de Tukey HSD. Sendo que os valores de A e C do grupo com uniao foram maiores que os do modelo mestre. (Tabela 3 - Alginato com união e modelo mestre).

Tabela 3. Alginato com união e modelo mestre- análise descritiva comparação entre grupos (em destaque: os valores de "p", aqui relatados pela sigla "sig" para cada medida A,B,C,D)

\begin{tabular}{|c|c|c|c|c|c|c|c|c|c|c|}
\hline & \multicolumn{2}{|c|}{$\begin{array}{l}\text { Levene's } \\
\text { Teest for } \\
\text { Equality } \\
\text { Variances }\end{array}$} & \multicolumn{7}{|c|}{$\mathrm{t}$-test for Equality of Means } \\
\hline & & F & sig. & $\mathrm{t}$ & df & Sig. & Mean Difference & 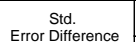 & $\begin{array}{l}95 \% \text { Confindence II } \\
\text { Difteren }\end{array}$ & vival of the \\
\hline & $\begin{array}{l}\text { Equal } \\
\text { varainces } \\
\text { acanced }\end{array}$ & 84,582 & ,000 & $|10,980|$ & 38 & .000 & .0141750 & ,0012910 & $\begin{array}{l}R_{0}, 0115616 \\
\end{array}$ & ,0167884 \\
\hline$A_{1}$ & $\begin{array}{l}\text { Equal } \\
\text { variances not }\end{array}$ & & & 10,980 & 19,000 & ,000 & .0141750 & ,0012910 & , 0114730 & . 0168770 \\
\hline & $\begin{array}{l}\text { Equalal } \\
\text { variances } \\
\text { vasumes }\end{array}$ & 42,348 & ,000 & \begin{tabular}{|l|}
, 046 \\
\end{tabular} & 38 & .302 & .0003450 & , 0003300 & - & . 0010130 \\
\hline B & 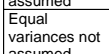 & & & 1,046 & 19,000 & .309 & .0003450 & , 0003300 & . & .0010356 \\
\hline & $\begin{array}{l}\text { Equalal } \\
\text { variances }\end{array}$ & 69,040 & ,000 & 2,490 & 38 & .017 & .0028825 & ,0011578 & , 0005387 & .0052263 \\
\hline c & \begin{tabular}{|l}
$\begin{array}{l}\text { Equal } \\
\text { variances not } \\
\text { assumed }\end{array}$ \\
\end{tabular} & & & 2,490 & $\mid 19,000$ & .022 & .0028825 & ,0011578 & , 0004593 & .053057 \\
\hline & 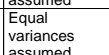 & 32,591 & ,000 & 1,157 & 38 & 254 & .0004650 & ,0004018 & -0003484 & .0012784 \\
\hline D & \begin{tabular}{|l} 
Earalan \\
assunes not not \\
asumed
\end{tabular} & & & 1,157 & $\mid 19,000$ & 261 & .0004650 & ,0004018 & - & .0013060 \\
\hline
\end{tabular}

Os dados estatísticos obtidos podem ser interpretados como a existência de diferenças significativas entre as medidas dos grupos com e sem a união dos análogos quando comparadas ao modelo mestre, não havendo medidas semelhantes entre os grupos com e sem a união. Sendo que sem a união houve alteração significativa nas medidas $\mathrm{A}, \mathrm{C}$ e $\mathrm{D}$ quando comparadas ao modelo mestre. E no grupo com a união houve alteração significativa nas medidas $\mathrm{A}$ e $\mathrm{C}$ quando comparadas ao modelo mestre.

\section{DISCUSSÃO}

A atenção aos conhecimentos atuais de oclusão na execução das atividades clínicas e laboratoriais é fundamental para devolver função, estética, fonética e autoestima aos pacientes ${ }^{9}$. A longevidade das fixações das estruturas suportadas por implantes depende do mínimo de desajuste possível, uma vez que a evidência científica mostrou que o assentamento passivo absoluto não pode ser alcançado. $\mathrm{O}$ perfil da prótese deve corresponder a toda conformação que se insere ${ }^{10}$.

Os implantes apresentam uma biomecânica mais suscetível à sobrecarga oclusal, concentrada na região de crista do rebordo ósseo onde se insere, devendo observar fatores como: qualidade óssea, direção do estresse aplicado sobre a prótese implanto-suportada, contatos prematuros e hábitos parafuncionais ${ }^{9}$. Assim também, o uso de técnicas incorretas pode gerar dificuldade em adquirir uma relação passiva entre prótese e implante, comprometendo a fixação dos parafusos. Algumas manobras para eliminar o desajuste e problemas associados ao torque incluem a manipulação da prótese ajustando-a através de secções e soldagens ${ }^{10}$. Aumentando muitas vezes o custo e tempo de trabalho, quando um bom planejamento clínico/laboratorial previne esse tipo de falha.

Para moldagens em implantodontia, a transferência da posição dos implantes ou componentes protéticos para os modelos de trabalho é feita através de técnicas como a de arrasto (moldeira aberta) com a união dos transferentes quadrados por resina acrílica que demonstra grande precisão, entretanto a contração de polimerização da resina pode gerar distorções $^{11,12}$. Para isso, essa pesquisa fez uso de um fio metálico rígido auxiliando na manutenção da posição correta dos transferentes.

Entre os materiais de moldagem, destacam-se os silicones por adição e os poliéteres, devido à alta precisão de suas impressões ${ }^{2,12}$. Já o alginato é um material de baixo custo quando comparado, fácil manipulação e boa precisão, podendo substituí-los em algumas situações ${ }^{13}$. Sendo utilizado comumente em moldagens preliminares ${ }^{14}$. Demonstrando-se nessa pesquisa um material adequado para uso em implantodontia.

Outro fator importante é a qualidade do gesso utilizado na produção dos modelos. A expansão de presa do gesso pode levar a imprecisões no planejamento e execução laboratorial, sendo bastante influenciada pela quantidade de água e composição química do gesso ${ }^{15}$. Os fabricantes recomendam que, para obtenção de valores bem próximos da ausência de expansão, devem-se seguir criteriosamente as especificações de seus produtos, observando rigorosamente à proporção água/pó. Porém existem algumas variáveis que também devem ser observadas, como temperatura e composição da água $^{16}$. Nesse trabalho pode-se observar que as condições favoráveis descritas pelo fabricante nem sempre são alcançadas na prática clínica e laboratorial, devido aos inúmeros fatores a serem controlados, que muitas vezes fogem do domínio do cirurgião-dentista.

Em um estudo com 3 tipos de gesso odontológico os valores médios ultrapassaram a especificação ${ }^{\circ} 25$ da $\mathrm{ADA}^{17}$, sendo a expansão de presa do gesso tipo IV menor que os gessos tipo III e $\mathrm{V}^{8}$. Comprovado pelos resultados obtidos nessa pesquisa que mostraram que as propriedades do gesso 
podem gerar alterações significativas nos trabalhos finais.

\section{CONCLUSÃO}

Com base na metodologia empregada e nos resultados obtidos concluiu-se que as técnicas com ou sem a união de análogos por fio metálico rígido e resina autopolimerizável apresentaram medidas estatisticamente diferentes quando comparadas ao modelo mestre. Sendo que a união de análogos gerou modelos com menores distorções quando comparadas ao modelo mestre. Conclui-se também que o alginato apresenta eficiência para moldagem em implantodontia.

\section{REFERÊNCIAS}

1. Matiello CN, Trentin MS. Implante dentário com carga imediata na região anterior superior: relato de caso clínico. RFO UPF. 2015; 20(2): 238-42.

2. Gomes EA, Assunção WG, Costa PS, Delben JA, Barão VAR. Moldagem de transferência de próteses sobre implante ao alcance do clínico geral. Pesq Bras Odontoped Clin Integr. 2006; 6(3):281-8.

3. Zuim PRJ, Sousa V, Garcia AR, Pellizzer EP, Rocha EP. Alterações dimensionais em modelos de gesso na moldagem de transferência em relação à esplintagem dos tranferentes, material de moldagem e paralelismo de implantes. Rev Odontol UNESP. 2002; 31(1):25-37.

4. Pieralini ARF, Lazarin AA, Segalla JCM, Silva RHBT da, Pinelli LAP. Aspectos periodontais do paciente idoso. Salusvita. 2008; 27(2):309-18.

5. Pavarina AC, Bussadori CMC, Cucci ALM, Vergani CE, Giampaolo ET. Influência da desinfecção de moldes na alteração dimensional de modelos de gesso. Rev Odontol UNESP. 1998; 27(2):381-91.

6. Valle AL do, Coelho AB, Scolaro JM. Avaliação do comportamento morfodimensional de materiais de moldagem utilizados em implantes dentais. Rev FOB. 2001; 9(1/2):41-8.

7. Pereira T, Santos Júnior GC, Rubo JH, Conti PCR. Avaliação da influência da alteração dimensional do gesso tipo IV nas moldagens de Implantodontia. RPG Rev Pós Grad. 2005; 12(1):43-50.

8. Ferreira AR, Nagem Filho H, Pinto JHN. Determinação da magnitude de expansão de alguns tipos de gesso. Salusvita. 2000; 19(2):29-39.

9. Sanitá PV, Pinelli LAP, Silva RHBT da, Segalla JCM. Aplicação clínica dos conceitos oclusais na implantodontia. RFO. 2009; 14(3):268-75.

10. Campi Junior L, Nagem Filho H, Fares NH, Missaka R, Fiuza CT, D'Azevedo MTFS. Passividade da prótese sobre implante. Innov Implant J, Biomater Esthet. 2010; 5(3):53-9.

11. Del'Acqua MA, Arioli Filho JN, Compagnoni MA, Mollo Júnior FA. Accuracy of impression and pouring techniques for an implant-supported prosthesis. Int J Oral Maxillofac Implants. 2008; 23(2):226-36.

12. Silva MM, Mima EGO, Del'Acqua MA, Segalla JCM, Silva RHBT, Pinelli LAP. Técnicas de moldagem em prótese sobre implantes. Rev Odontol UNESP. 2008; 37(4):301-8.

13. Monteiro WC, Corradi CAL, Consani S, Mendes WB. Dimensional changes occured in cast for complete dentures evaluated by tri-dimensional measurements. Cienc Odontol Bras. 2007; 10(3):6-11.

14. Gennari Filho H, Assunção WG, Rocha EP, Goiato MC. Avaliação da eficácia de alguns materiais de moldagem na reprodução do posicionamento de implantes com inclinações variáveis, para obtenção de modelos preliminares. ROBRAC. 2003; 12(33):34-9.

15. Lopes LAZ, Cezero L, Mezzomo E, Suzuki RM. Precisão na montagem em articulador em função do tipo de gesso e tratamento do modelo. Cienc Odontol Bras. 2003; 6(2):65-71.

16. Sansiviero A, Bauer JRO, Masuda MS, Sigemori RM, Oliveira MT de, Tavares Junior CARF et al. Expansão de presa de gesso tipo IV (pedra) de alta resistência em diferentes técnicas de manipulação e marcas diversas. ConScientiae Saúde. 2008; 7(3):373-8.

17. American Dental Association. Guide to dental materials and devices. 6. ed., Chicago, 1972/1973, p.253-858, (specification $\mathrm{n}^{\circ} 25$ for dental gypsun products).

\section{CONFLITO DE INTERESSES}

Os autores declaram não haver conflitos de interesse.

\section{AUTOR PARA CORRESPONDÊNCIA}

\section{Nelson Talatoci Oshiro Filho}

nelsonoshirof@hotmail.com

Submetido em 23/02/2018

Aceito em 27/03/2018 